\title{
Stripping Material from a Supported Lipid Bilayer with High Speed Buffer Flow
}

\author{
Michael J. Ornstead, Ruth Hunter, Mason L. Valentine, Cameron Cooper, Stephen K. Smith, \& Christopher F. Monson* \\ Department of Physical Science, Southern Utab University, Cedar City, UT \\ bttps:/ / doi.org/10.33697/ ajur.2020.027 \\ Students:mikeornstead@yahoo.com,kruth.hunter@gmail.com,masonlvalentine@utexas.edu,cam_cooper90@hotmail.com, \\ stephenksmith263@gmail.com \\ Mentor: christophermonson1@suu.edu
}

\begin{abstract}
A microfluidic device was created and used to demonstrate that supported lipid bilayers can be deposited on clean glass slides and removed using high velocity buffer flow (1-4 m/s linear velocity). This was accomplished by forcing the flow through a microfluidic channel covering an annealed glass coverslip bearing a supported lipid bilayer (SLB). The removal of bilayer material was monitored via fluorescence microscopy, and two basic regimes were observed: at 1-2 m/s smaller areas were stripped, while at $3-4 \mathrm{~m} / \mathrm{s}$ larger areas were stripped. SLB removal was verified by two means. First, lipid vesicles labeled with a different fluorescent dye were added to the device and filled in holes left by the removal of the original SLB, allowing stripping to be verified visually. Second, the solutions obtained from stripping were concentrated and the fluorescence in the concentrates was measured. The ability to strip SLB from glass provides a relatively gentle method of creating spatially inhomogeneous SLB, which could be a useful tool in the continued investigation of membrane properties and components.
\end{abstract}

\section{KEYWORDS}

Supported Lipid Bilayer; Membrane Vesicle; Microfluidic Device

\section{INTRODUCTION}

A supported lipid bilayer (SLB) consists of two leaflets of amphiphilic molecules, typically phospholipids, supported by a clean, flat substrate, often glass. ${ }^{1,2}$ SLBs retain many of the properties of biological lipid membranes such as those found in cells. ${ }^{3}$ Molecules in a SLB retain their lateral fluidity, and the amphiphilic nature of SLBs causes molecules to assume configurations similar to those assumed in native cell membranes, thus they are of interest as native membrane mimics. ${ }^{4-7}$ Most SLBs are composed primarily of phospholipids, which have a hydrophilic head and a hydrophobic tail, but other, similarly structured lipids can also be used, and molecules like cholesterol can be added to modify the properties of the bilayer. ${ }^{8-11}$

SLBs can be patterned on their substrates using a variety of methods. For example, barriers can be placed photolithographically or by other means to control SLB formation. ${ }^{12-16}$ Alternatively, patterns can be created after a bilayer is formed, for example by scratching to create a barrier, applying an electric field to induce movement of charged lipids or proteins, using DNA or microbeads. ${ }^{17-23}$ Patterns in SLBs can also be created using polymer stencils, applied either before a bilayer is deposited or used after the bilayer has been deposited to lift off a section of bilayer. ${ }^{24,25}$ Such patterns can be used to facilitate the purification of membrane components.

One of the principal uses for SLBs is as model cell membranes, particularly in the study of membrane proteins. Membrane proteins are integral to many cell functions including signaling, metabolism, and cell structure. ${ }^{26}$ Despite their importance, membrane proteins are challenging to work with due to their amphiphilic nature - most membrane proteins are only stable and active in a lipid bilayer. ${ }^{27-29}$ Despite these challenges, there are numerous examples of work that has been done with membrane proteins and peptides, including spectroscopy, ${ }^{30}$ crystal structure, ${ }^{31}$ and membrane organization. ${ }^{32}$ Several membrane protein methods have been developed that use supported lipid bilayers. ${ }^{32-36}$

Among SLB techniques that show great promise with membrane proteins are those that involve separations. Several separation methods have been developed that rely on lipid and protein motion in electric fields, including line electrophoresis and electrophoretic-electroosmotic flow. ${ }^{37-40}$ In other separation methods bulk flow, ${ }^{41-42}$ lipid curvature, ${ }^{43}$ Brownian motion, ${ }^{44}$ or surface acoustic waves ${ }^{45}$ are used to sort membrane components. To allow work with membrane proteins, these techniques can be combined with molecular cushions, which minimize protein denaturation on the support surface. ${ }^{33,34,46-52}$ 
While these techniques show great promise, particularly in combination, they have limitations with certain proteins, particularly proteins that transport large molecules, as the space on the supported side of the bilayer is insufficient to allow for the transport of a large molecule. We introduce a method of removing SLB components in an effort to create vesicles of materials after, for example, they have been purified by SLB-based techniques. Other methods have been developed to remove SLB components, but they typically use detergents and thus would remove any proteins from the membrane environment. ${ }^{53}$ Another method growing in popularity uses a mixture of styrene and maleic acid, which is less denaturing for proteins. ${ }^{54,55}$ Our method involves high-speed buffer flow, as will be discussed below.

\section{MATERIALS AND PROCEDURES}

\section{Lipid vesicle preparation}

Lipid vesicles were prepared using a modified version of the freeze-thaw extrusion method. ${ }^{56-57}$ Dried lipids were dissolved in chloroform and mixed in appropriate volumes to produce the desired lipid compositions (1\% fluorophore labeled lipid in 1palmitoyl-2-oleoyl-glycero-3-phosphocholine (POPC), Avanti Polar Lipids, Alabaster, Alabama). The fluorophores used were fluorescein-labeled 1,2-dioleoyl-sn-glycero-3-phosphoethanolamine (Fl-DOPE, Avanti Polar Lipids, Alabaster, Alabama) and Texas Red-labeled 1,2-dihexadecanoyl-sn-glycero-3-phosphoethanolamine (TR-DHPE, Biotium, Inc., Fremont, California). The chloroform was evaporated in a stream of air and then in a vacuum at 3 torr for at least 4 hours. The dried lipids were hydrated with a tris(hydroxymethyl)aminomethane (Tris, Fisher Scientific) buffer solution (0.1 M NaCl, $0.02 \mathrm{M}$ Tris, pH 7.4) while being continuously but gently swirled. The vesicles were then extruded using a mini-extruder (Avanti Polar Lipids, Alabaster, Alabama) with a $100 \mathrm{~nm}$ pore track-etched membrane (Whatman, Maidstone, United Kingdom). The solution was forced through the filter ten times in each direction, for twenty total passes, and then stored at $4{ }^{\circ} \mathrm{C}$ until use.

\section{Glass Cleaning}

Glass coverslips (24 x $50 \mathrm{~mm}$, Fischer) were cleaned by boiling in 7x detergent solution (MP Biomedical) at around 1:10 dilution with water for an hour, followed by rinsing with deionized water $(18 \mathrm{M} \Omega)$ for several minutes. The slides were annealed at $530^{\circ} \mathrm{C}$ for several hours and then stored in a clean slide box until use. ${ }^{58-59}$

\section{Device Fabrication}

Microfluidic devices were fabricated individually in a multi-step process. A box was first constructed out of glass using superglue to bond pieces together. The bottom glass piece had two 8 x $8 \mathrm{~mm} 150 \mu \mathrm{m}$ thick glass pieces glued near its center, about $10 \mathrm{~mm}$ apart. These were the "landing pads." A second piece of glass, about $4 \mathrm{~mm}$ wide and $12 \mathrm{~mm}$ long, was placed between these two glass pieces (but not glued to them), held off the base by the landing pads. Enough polydimethylsiloxane (PDMS, Silgard 184, Dow Corning) to just cover the landing pads was then poured into the box, and the device was baked at $110{ }^{\circ} \mathrm{C}$ for $10 \mathrm{~min}$. Metal wire was used to create a junction between the micro-scale portion of the device and the supply tubes - the wire was carefully placed such that it ended over the landing pads and extended to the outer edge of the box. Another $\sim 2$ mm layer of PDMS was then added to the device and hardened at $110^{\circ} \mathrm{C}$ for $10 \mathrm{~min}$. Pieces of $1 \mathrm{~mm}$ thick glass slides were then added to the device as internal stiffeners, and an additional $\sim 3 \mathrm{~mm}$ layer of PDMS was added. Another two layers of stiffening glass in $\sim 2 \mathrm{~mm}$ each of PDMS (four mm total) were baked in the device, and a final layer of PDMS was poured over the device until the device height was around $1.5 \mathrm{~cm}$, at which point the device was baked overnight. The glass box was then removed, which also removed the landing pads. Two exacto knife blades were taped together and used to cut a straight channel connecting the two landing pads, as shown in Figure 1.

\section{Stripping Procedure}

A clean glass coverslip was clamped to the device with a $1 \mathrm{~mm}$ thick glass slide behind it for additional strength, and the device was soaked in DI water in a vacuum jar at 10 torr for at least 30 minutes to remove air from the device. The device was then loaded with $5 \mathrm{mM}$ Tris (no NaCl, pH 7.5) buffer solution, following which it was loaded with 60 microliters of 1\% TR-DHPE or $1 \%$ Fl-DOPE lipid vesicles (in POPC and Tris). Following loading, the device was allowed to sit for at least 30 minutes to allow for the formation of a supported lipid bilayer (SLB). The device was then rinsed with $5 \mathrm{mM}$ Tris buffer solution at a rate of 0.2 $\mathrm{m} / \mathrm{s}$, for at least 10 minutes, in order to remove excess vesicles from the device. The flow rate was then increased to between 1.1 and $4.2 \mathrm{~m} / \mathrm{s}$ to strip material from the SLB. Stripping in the channel was monitored via fluorescence microscopy.

\section{Backfilling Procedure}

Following a successful stripping attempt, the device was loaded with 60 microliters of the fluorescent lipid not used initially (TRDHPE or Fl-DOPE) in POPC and allowed to sit for at least five minutes for backfilling to occur. The device was then rinsed with $5 \mathrm{mM}$ Tris buffer at $0.5 \mathrm{~mL} / \mathrm{min}$ to remove excess vesicles from the device, and backfilling was evaluated using fluorescence microscopy. 

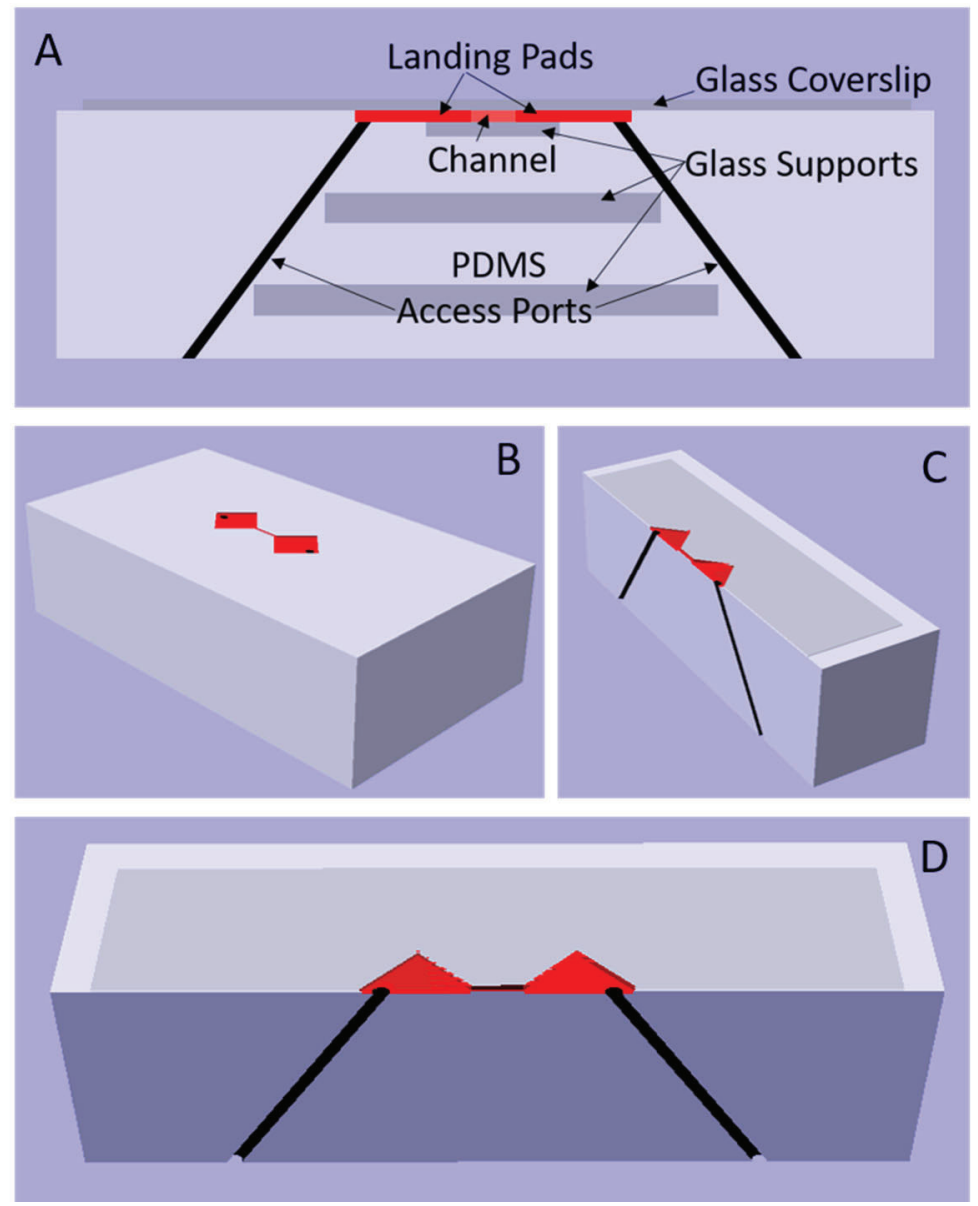

Figure 1. Device illustration. Panel A shows a labeled cutaway side view of the device. The bilayer is localized to the landing pads and the channel (attached to the removable glass coverslip). The access ports carry solution to and from the bilayer. There is a piece of glass coverslip at the bottom of the channel, and four $1 \mathrm{~mm}$ thick pieces of glass slides inside the device (only two are shown, the other two are between the two shown and are on either side of the access ports). B shows the entire device without a coverslip, and $\mathrm{C}$ and $\mathrm{D}$ show cutaway perspective views of the device with a coverslip.

\section{RESULTS}

The SLBs initially appeared smooth after rinsing as expected (Figure 2, 0 min). In Figure 2, the bright area is the fluorescently labeled lipid bilayer (labeled with 1\% TR-DHPE in POPC). The areas that are initially dark are those areas of glass bonded to the PDMS device and that thus bear no lipids. After solution was flowed over the bilayer at a high speed, dark defects started to occur, often in a linear pattern (Figure 2, 5 min). These defects would commonly grow with time. To verify that the defects were not due to an elastic lateral compression of the lipids, the defects were observed both while buffer was flowing and then with no flow, and no change was observed. The defects (darker areas appearing in the light areas) are at least in part due to lipids being
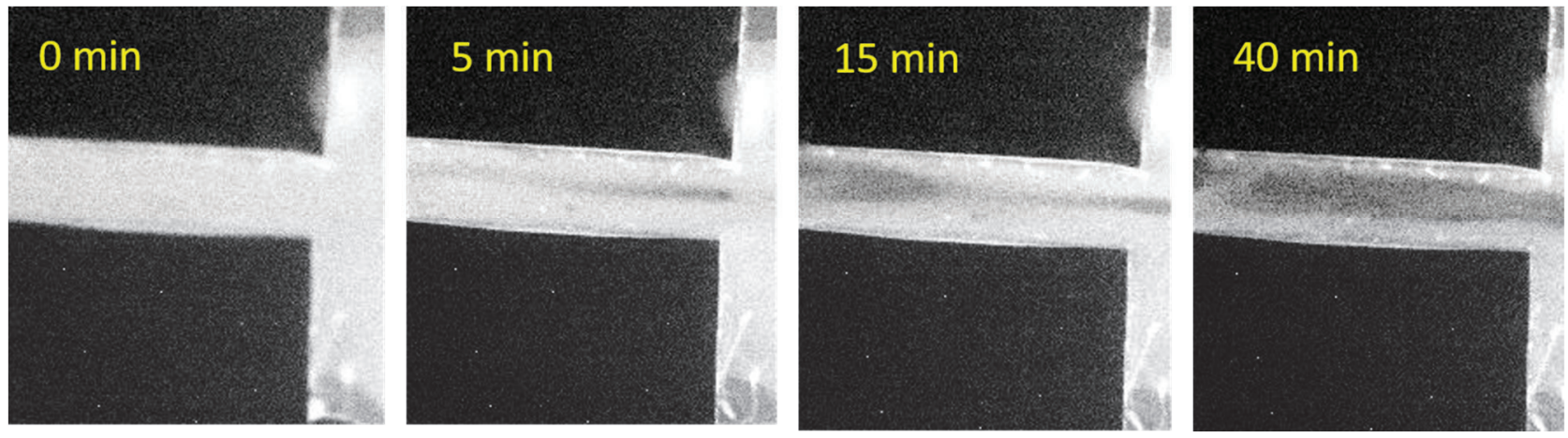

Figure 2. Stripping a SLB from a glass surface as seen by fluorescence microscopy. As can be seen, an initially smooth bilayer is removed from much of the central channel area over the course of 40 minutes at a flow rate of around $1.1 \mathrm{~m} / \mathrm{s}$. 
stripped from the solid support and returned to the aqueous phase (referred to hereafter as eluent, in analogy to chromatography), presumably as micelles or vesicles.

As the stripped area was observed to increase with time and the rate of stripping was observed to depend on the flow rate, the efficiency of various flow rates could be compared, as shown in Figure 3. As might be expected, while SLB stripping occurred at both slower and faster flow rates, the efficiency of the stripping differed. At low flow rates $(1-2 \mathrm{~m} / \mathrm{s}$ linear flow rate, or 3-4 $\mathrm{mL} / \mathrm{min}$ volumetric flow rate), the stripping happened more slowly (taking on average 40 minutes to strip the maximum area that could be stripped - additional time did not result in further bilayer stripping) but the efficiency in terms of volume of eluent used and thus bilayer material concentration in the eluent was maximized. On the other hand, at high flow rates $(\sim 4 \mathrm{~m} / \mathrm{s}$ linear flow rate or $10 \mathrm{~mL} / \mathrm{min}$ volumetric flow rate) the stripping was faster, although it used somewhat more eluent. Most of the initial runs were performed at low flow rates, while later runs were typically at higher flow rates. It should also be noted that higher flow rates subjected the device to significant stress and that device failure was more common at higher flow rates. We also tested solutions of different ionic strengths: from $1 \mathrm{M} \mathrm{NaCl} / 0.01 \mathrm{M}$ Tris to deionized water, and did not observe any appreciable effects on stripping time, area, or efficiency.
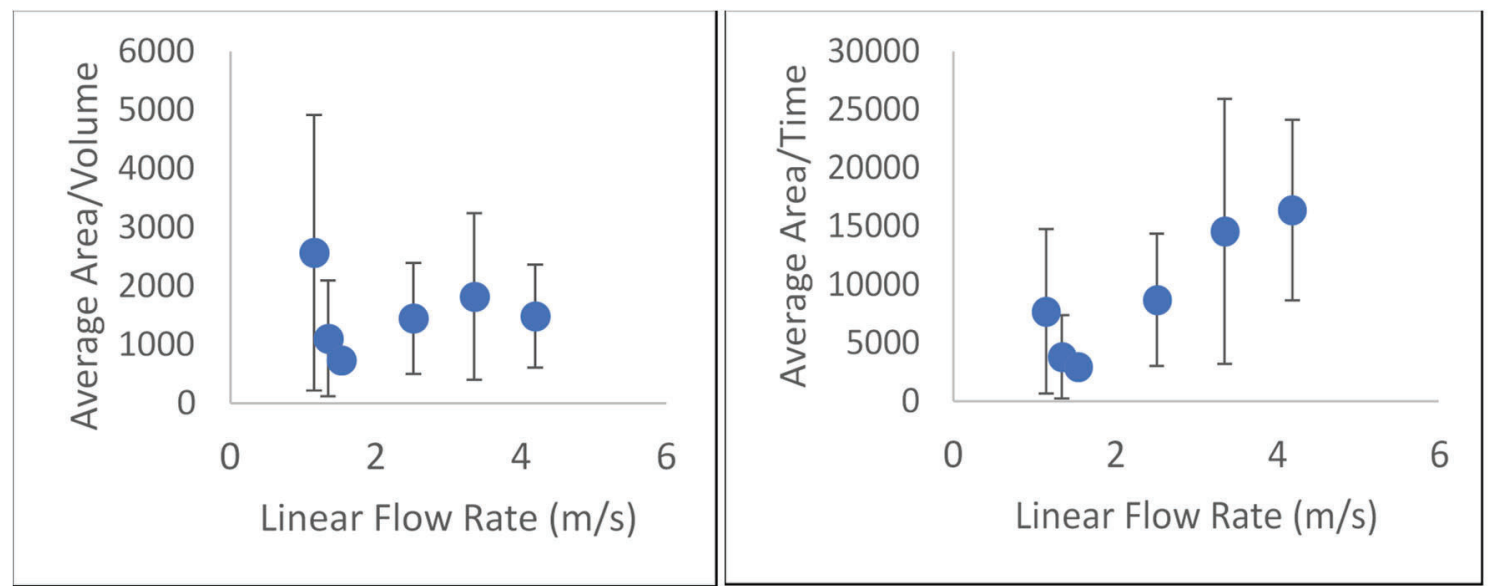

Figure 3. Stripping efficiency as a function of flow rate. Stripping efficiency is illustrated as the average area stripped per volume of solution (in $\mu \mathrm{m}^{2} / \mathrm{mL}$ ) and average area stripped per time (in $\mu \mathrm{m}^{2} / \mathrm{min}$ ), both as a function of linear flow rate of buffer over the SLB (in $\mathrm{m} / \mathrm{s}$ ). As can be seen in the graphs, the stripping is slightly more efficient per volume (resulting in a higher concentration of stripped lipids) at lower flow rates but more time efficient at higher flow rates. Error bars are standard deviations.

To verify that the observed darkening was indeed lipid loss to the eluent as opposed to lipid migration or another analogous process, several experiments were performed. First, additional lipids labeled with a different fluorophore were added after a stripping run was performed. If the lipids initially on the glass had been completely removed by the solution flow, the bare glass surface should induce bilayer reformation with the new vesicles. Thus stripping runs were performed on a 1\% Fl-DOPE in POPC SLB and then 1\% TR-DHPE in POPC vesicles were added to the stripped bilayer. This is illustrated in Figure 4. The bilayer initially is an even green (due to the Fl-DOPE). After the stripping, there are dark regions in the bilayer where the initial bilayer has been stripped away (Figure 4 B). After TR-DHPE labeled lipids are added, many of these dark areas are observed to fluoresce red, demonstrating that the TR-DHPE labeled lipids have formed a bilayer on the glass.

This method of removing and backfilling lipids may be useful for more than just demonstrating that stripping has occurred. The commonly employed methods for patterning bilayers when two patches of contiguous bilayer are required to be different is either to put one bilayer down, scratch to remove part of the bilayer, and then backfill the scratched region with additional lipids ${ }^{37}$ or to use polymer blockers to prevent a region from being filled with lipids and then remove the blockers and fill the space with a second type of lipid. ${ }^{60}$ Using high speed buffer flow to remove SLB should be more gentle than scratching the surface ${ }^{37}$ and not potentially leave a residue like polymer blockers. ${ }^{60}$ Thus, it could be a more effective way to create an SLB with two compositionally distinct regions. We attempted to determine whether this would be the case by looking at fluorophore-labeled lipid diffusion from one region of the bilayer to the other, however our results were inconclusive - it appeared that some regions demonstrated diffusion, but not all. 

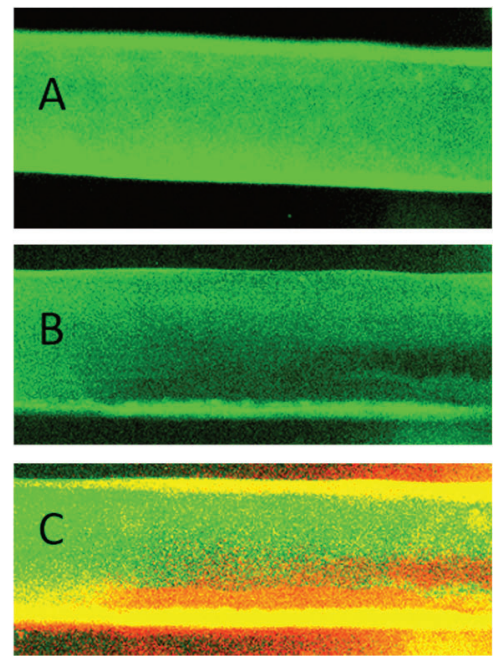

Figure 4. Stripping and backfilling lipids. A shows the initial bilayer (before stripping takes place). B shows the bilayer after stripping has taken place but before lipids have been backfilled. C shows the bilayer after stripping and backfilling. The lipids present initially were Fl-DOPE labeled (shown green) and the bilayer was backfilled with TR-DHPE labeled (shown red). Notice that the TR-DHPE lipids are most concentrated where the Fl-DOPE lipids have been stripped.

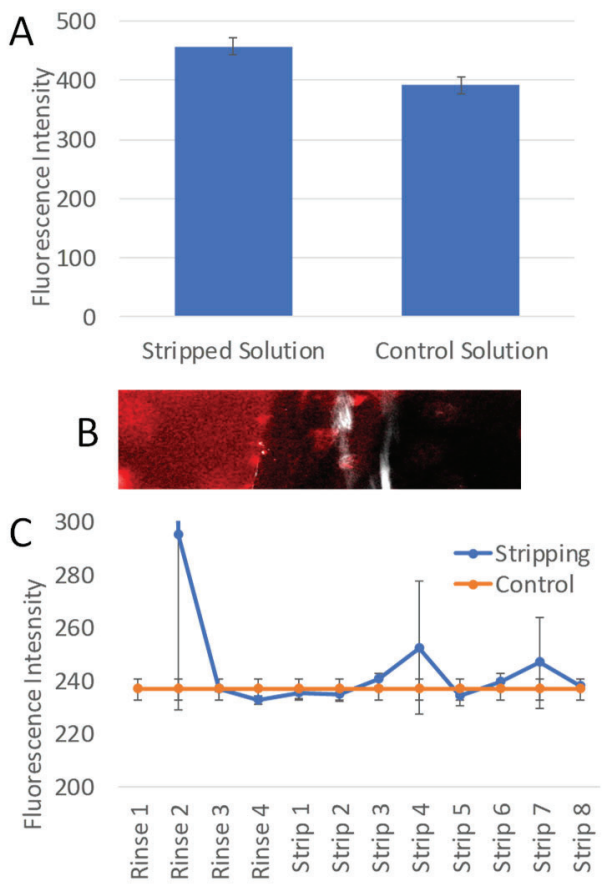

Figure 5. The fluorescence of the solution used to strip a bilayer from a solution compared to the fluorescence of a solution passed through the microfluidic device but with no fluorescent lipids present. A: the average fluorescence observed over 10 measurements for the stripped solution and the control solution. B: an image of the fluorescence observed with a fluorescence microscope of the stripped solution (left) a PDMS divider (center) and the control solution (right). C: observed fluorescence divided into time steps. The initial peak is due to unbound vesicles being rinsed away.

To verify that the lipids that were being removed were not simply migrating to other places in the device, the eluent used to strip a run was collected (not including the initial rinse, which was discarded) and compared to solution run through the device with no bilayer at identical flow rates and times. For each solution, around $0.02 \mathrm{~g}$ of SDS was added to the solution as it was being collected to ensure any lipids remained in solution. While no fluorescence was observed in either solution initially (which was expected, as the fluorescent lipids were highly diluted), upon concentration by evaporation (from $\sim 300 \mathrm{~mL}$ to $3 \mathrm{~mL}$ ) the stripped 
solution exhibited significantly greater fluorescence than the control solution (which was indistinguishable from noise, the difference was $>99.9 \%$ confidence level as determined by a t-test). These results are shown in Figure 5.

Finally, we feared that these results might be due to incomplete rinsing. To demonstrate that this was not the case, we collected the solution passed through the microfluidic device into aliquots of around $1.5 \mathrm{~mL}$ for rinsing and $30 \mathrm{~mL}$ for stripping. As before, around $0.02 \mathrm{~g}$ of SDS was added to each aliquot and then the aliquots were evaporated down to $1 \mathrm{~mL}$ and the fluorescence of each aliquot tested. The results are shown in Figure 5 C. As was expected, the fluorescence signal for the first two rinsing steps when lipids were used was much larger than any other signal (the first step fluorescence is so large that it is not shown, $>2000$ units), as in this step excess lipids (lipids that did not stick to the glass slide) were being rinsed away. By the third and fourth rinsing aliquots, the fluorescence signal was essentially indistinguishable from the control (which was noise-limited). When the flow rate was increased to induce stripping, the fluorescence was observed to increase with additional peaks in the fluorescence that at least roughly correlated to observed stripping events. It should be noted that this stripping occurred at different times in each run and Figure $5 \mathrm{C}$ shows the averaged results of three runs, thus none of the peaks shown are statistically different (as determined by a t-test, the greatest confidence level is at $70 \%$ ) than the control as a given peak typically only occurred in one of the three runs. However, on an individual run the difference between a peak and the control was significant $(>99 \%$ confidence level via t-test).

\section{DISCUSSION}

Others have previously obtained results suggesting that high velocity flow above a bilayer can cause changes to a bilayer, ${ }^{41,42}$ and that lipid material can be removed from a bilayer during the formation of the bilayer, ${ }^{59}$ but to our knowledge this is the first report of high velocity flow moving SLB material into the aqueous solution without the aid of detergents. While our experiments were performed exclusively with lipids, this raises the possibility that such experiments could also be performed with membrane proteins contained in an SLB. This would be particularly valuable as a step following an SLB-based protein separation step, such as EOF, ${ }^{40}$ particularly if the removed SLB material forms vesicles as it is stripped from the surface. In lipid-only experiments, we attempted to determine the size of material coming off the supported lipid bilayer to determine if vesicle-like material or micellelike material was being generated, but the material generated was too dilute to be detected in our particle sizer.

In all experiments performed, it appeared that the stripping of materials from the support was not perfect - while significant sections of the stripped area have their lipids removed, small pockets of the original bilayer remain even in the stripped regions. In a set of stripping followed by backfilling experiments not shown, texas red-labeled lipid was stripped and the sections were backfilled with fluorescein (the reverse of the experiment shown in Figure 4). In this case, the stripping caused dark patches to appear, but when backfilling was attempted the increase in fluorescein fluorescence in the dark patch was relatively small. This is attributed to the FRET quenching of the fluorescein by remaining texas red-labeled lipid. This interpretation is also implied by the fact that only very rarely do the stripped regions get as dark as the lipid-free regions (e.g. in Figure 2, compare the stripped regions to the regions with no bilayer due to PDMS protection).

\section{CONCLUSIONS}

We have demonstrated material can be removed from a supported lipid bilayer by high-speed buffer flow over the bilayer. This was accomplished inside of a microfluidic device by flowing solution over the SLB at flow rates of from 1 to around $4 \mathrm{~m} / \mathrm{s}$ linear flow rate. Stripping of material from the supported lipid bilayer was observed to take place at flow rates greater than $1 \mathrm{~m} / \mathrm{s}$ and with all aqueous solutions tested. Stripping was demonstrated both by observing lipid loss from the bilayer using fluorescence microscopy and by monitoring fluorescence in the solution used to strip the material from the bilayer. Our results suggest that, if previously developed bilayer-based techniques were used to purify proteins in an SLB, ${ }^{38-45}$ the method we report in this paper could then be used to generate solution-phase purified proteins and lipids. This could be valuable for further biochemical work, and we are developing a device to attempt this. Alternatively, stripping SLBs using high speed buffer flow could be used to generate SLBs with compositionally distinct regions.

\section{ACKNOWLEDGEMENTS}

The authors thank Professor Susan Harris for making the ceramic rack used to anneal our glass coverslips. We thank Joshua A. Reid and Whitney N. Hansen for their work on this project. This project was funded by Southern Utah University's Faculty Scholarly Support Fund, undergraduate research grants, and Skaggs endowment grants. C. C., M. V., and S. S. wish to thank the Walter Maxwell Gibson endowment for research fellowships.

\section{REFERENCES}

1. Johnson, S. J., Bayerl, T. M., McDermott, D. C., Adam, G. W., Rennie, A. R., Thomas, R. K., Sackmann, E. (1991) Structure of an Adsorbed Dimyristoylphosphatidylcholine Bilayer Measured with Specular Reflection of Neutrons, Biophys J 59, 289294. https:// doi.org/10.1016/S0006-3495(91)82222-6 
2. Tamm, L. K., McConnell, H. M. (1985) Supported Phospholipid Bilayers, Biophys J 47, 105-113. https:// doi.org/ 10.1016/S0006-3495(85)83882-0

3. Smith, B. A., McConnell, H. M. (1978) Determination of Molecular Motion in Membranes Using Periodic Pattern Photobleaching, Proc Natl Acad Sci USA 75, 2759-2763. bttps:/ / doi.org/10.1073/pnas.75.6.2759

4. Kurniawan, J., Ventrici de Souza, J. F., Dang, A. T., Liu, G.-Y., Kuhl, T. L. (2018) Preparation and Characterization of SolidSupported Lipid Bilayers Formed by Langmuir-Blodgett Deposition: A Tutorial, Langmuir 34, 15622-15639. https:/ / doi.org/ 10.1021 / acs.langmuir.8603504

5. Castellana, E. T., Cremer, P. S. (2006) Solid Supported Lipid Bilayers: From Biophysical Studies to Sensor Design, Surf Sci Rep 61, 429-444. https:/ / doi.org/10.1016/j.surfrep.2006.06.001

6. Phizicky, E., Bastiaens, P. I. H., Zhu, H., Snyder, M., Fields, S. (2003) Protein analysis on a proteomic scale, Nature 422, 208215. https:// doi.org/10.1038/ nature01512

7. Zhu, H., Bilgin, M., Bangham, R., Hall, D., Casamayor, A., Bertone, P., Lan, N., Jansen, R., Bidlingmaier, S., Houfek, T., Mitchell, T., Miller, P., Dean, R. A., Gerstein, M., Snyder, M. (2001) Global analysis of protein activities using proteome chips, Science 293, 2101-2105. https:// doi.org/10.1126/science.1062191

8. Sendecki, A. M., Poyton, M. F., Baxter, A. J., Yang, T., Cremer, P. S. (2017) Supported Lipid Bilayers with Phosphatidylethanolamine as the Major Component, Langmuir 33, 13423-13429. https:// doi.org/ 10.1021/acs.langmuir.7b02323

9. Veatch, S. L., Keller, S. L. (2005) Seeing Spots: Complex Phase Behavior in Simple Membranes, Biochim Biophys Acta 1746, 172-185. https:// doi.org/10.1016/j.bbamcr.2005.06.010

10. Rayermann, S. P., Rayermann, G. E., Cornell, C. E., Merz, A. J., Keller, S. L. (2017) Hallmarks of Reversible Separation of Living, Unperturbed Cell Membranes into Two Liquid Phases, Biophys J 113, 2425-2432. bttps:/ / doi.org/10.1016/j.bpj.2017.09.029

11. Tabaei, S. R., Jackman, J. A., Kim, S.-O., Liedberg, B., Knoll, W., Parikh, A. N., Cho, N.-J. (2014) Formation of CholesterolRich Supported Membranes Using Solvent-Assisted Lipid Self-Assembly, Langmuir 30, 13345-13352. https:// doi.org/10.1021/la5034433

12. Mossman, K. D., Campi, G., Groves, J. T., Dustin, M. L. (2005) Altered TCR Signaling from Geometrically Repatterned Immunological Synapses, Science 310, 1191-1193. https:/ / doi.org/10.1126/ science.1119238

13. Kung, L. A., Kam, L., Hovis, J. S., Boxer, S. G. (2007) Patterning Hybrid Surfaces of Proteins and Supported Lipid Bilayers, Langmuir 16, 6773-6776. bttps:/ / doi.org/10.1021/la000653t

14. Nair, P. M., Salaita, K., Petit, R. S., Groves, J. T. (2011) Using Patterned Supported Lipid Membranes to Investigate the Role of Receptor Organization in Intracellular Signaling, Nat Protoc 6, 523-539. https:// doi.org/ 10.1038/nprot.2011.302

15. Mossman, K., Groves J. (2007) Micropatterned Supported Membranes as Tools for Quantitative Studies of the Immunological Synapse, Chem Soc Rev 36, 46-54. https:// doi.org/10.1039/b605319j

16. Nabika, H., Sasaki, A., Takimoto, B., Sawai, Y., He, S., Murakoshi, K. (2005) Controlling Molecular Diffusion in SelfSpreading Lipid Bilayer Using Periodic Array of Ultra-Small Metallic Architecture on Solid Surface J Am Chem Soc 127, 16786-16787. https://doi.org/10.1021/ja0559597

17. Hughes, L. D., Boxer, S. G. (2013) DNA-Based Patterning of Tethered Membrane Patches, Langmuir 29, $12220-12227$. bttps://doi.org/10.1021/la402537p

18. Cremer, P. S., Boxer, S. G. (1999) Formation and Spreading of Lipid Bilayers on Planar Glass Supports, J Phys Chem 103, 2554-2559. https:// doi.org/10.1021/jp983996x

19. Groves, J. T., Boxer, S. G. (1995) Electric Field-Induced Concentration Gradients in Planar Supported Bilayers, Biophys J 69, 1972-1975. https://doi.org/10.1016/S0006-3495(95)80067-6

20. Groves, J. T., Wülfing, C., Boxer, S. G. (1996) Electrical Manipulation of Glycan-Phosphatidyl Inositol-Tethered Proteins in Planar Supported Bilayers, Biophys J 71, 2716-2723. https:/ / doi.org/ 10.1016/S0006-3495(96)79462-6

21. Groves, J. T., Boxer, S. G., McConnell, H. M. (1997) Electric field-induced reorganization of two-component supported bilayer membranes, Proc Natl Acad Sci USA 94, 13390-13395. https://doi.org/10.1073/pnas.94.25.13390

22. Grogan M. J., Kaizuka, Y., Conrad, R. M., Groves, J. T., Bertozzi, C. R. (2005) Synthesis of Lipidated Green Fluorescent Protein and its Incorporation in Supported Lipid Bilayers, J Am Chem Soc 127, 14383-14387. https://doi.org/10.1021/ja052407f

23. Bulloch, T. M., Argyle, T. C., Parson, M. G., Monson, C. F. (2016) Patterning Supported Lipid Bilayers Using Magnetic Tweezers, J Utah Acad 93, 269-281.

24. Zhu, Y., Moran-Mirabal, J. (2016) Micropatterning of Phase-Segregated Supported Lipid Bilayers and Binary Lipid Phases through Polymer Stencil Lift-Off, Langmuir 32, 11021-11028. https:/ / doi.org/ 10.1021/ acs.langmuir.6b02346

25. Richards, M. J., Daniel, S. (2018) Two-Phase Contiguous Supported Lipid Bilayer Model for Membrane Rafts via Polymer Blotting and Stenciling, Langmuir 33, 1285-1294. https:/ / doi.org/10.1021/ acs.langmuir.6b04385

26. Zhou, Y., Gao, J., Zhu, H., Xu, J., He, H., Gu, L., Wang, H., Chen, J., Ma, D., Zhou, H., Zheng, J. (2018) Enhancing Membrane Protein Identification Using a Simplified Centrifugation and Detergent-Based Membrane Extraction Approach, Anal Chem 90, 2434-2439. https:/ / doi.org/10.1021/acs.analchem.7b03710 
27. Santoni V., Molloy, M., Rabilloud, T. (2000) Membrane proteins and proteomics: Un amour impossible? Electrophor 21, 10541070. https:// doi.org/10.1002/(SICI)1522-2683(20000401)21:6<1054::AID-ELPS1054>3.0.CO;2-8

28. Braun R. J., Kinkl, N., Beer, M., Ueffing, M. (2007) Two-dimensional electrophoresis of membrane proteins, Anal Bioanal Chem 389, 1033-1045. bttps: / / doi.org/10.1007/s00216-007-1514-6

29. Rabilloud T. (2009) Membrane proteins and proteomics: love is possible, but so difficult, Electrophor 30 (S1), S174-S180. https:// doi.org/ 10.1002/ elps.200900050

30. Berweger, S., Nguyen, D. M., Muller, E. A., Bechtel, H. A., Perkins, T. T., Raschke, M. B. (2013) Nano-Chemical Infrared Imaging of Membrane Proteins in Lipid Bilayers J Am Chem Soc 135, 18292-18295. bttps:/ / doi.org/10.1021/ja409815g

31. Long, F., Su, C. C., Zimmermann, M. T., Boyken, S. E., Rajashankar, K. R., Jernigan, R. L., Yu, E. W. (2010) Crystal Structures of the CusA Efflux Pump Suggest Methionine-Mediated Metal Transport, Nature 467, 484-488. https:// doi.org/10.1038/nature09395

32. Sharma, V. K., Qian, S. (2019) Effect of an Antimicrobial Peptide on Lateral Segregation of Lipids: A Structure and Dynamics Study by Neutron Scattering, Langmuir 35, 4152-4160. https:// doi.org/10.1021/acs.langmuir.8b04158

33. Pace, H., Nyström L. S., Gunnarsson, A., Eck, E., Monson, C., Geschwindner, S., Snijder, A., Höök, F. (2015) Preserved Transmembrane Protein Mobility in Polymer-Supported Lipid Bilayers Derived from Cell Membranes, Anal Chem 87, 91949203. bttps://doi.org/10.1021/acs.analchem.5b01449

34. Davis, R. W., Flores, A., Barrick, T. A., Cox, J. M., Brozik, S. M., Lopez, G. P., Brozik, J. A. (2007) Nanoporous Microbead Supported Bilayers: Stability, Physical Characterization, and Incorporation of Functional Transmembrane Proteins, Langmuir 23, 3864-3872. https:// doi.org/10.1021/la062576t

35. Zieleniecki, J. L., Nagarajan, Y., Waters, S., Rongala, J., Thompson, V., Hrmova, M., Köper, I. (2016) Cell-Free Synthesis of a Functional Membrane Transporter into a Tethered Bilayer Lipid Membrane, Langmuir 32, 2445-2449. bttps:// doi.org/10.1021/ acs.langmuir.5b04059

36. Casiraghi, M., Damian, M., Lescop, E., Point, E., Moncoq, K., Morellet, N., Levy, D., Marie, J., Guittet, E., Banères, J.-L., Catoire, L. J. (2016) Functional Modulation of a G Protein-Coupled Receptor Conformational Landscape in a Lipid Bilayer, J Am Chem Soc 138, 11170-11175. https:// doi.org/10.1021/jacs.6b04432

37. Daniel, S., Diaz, A. J., Martinez, K. M., Bench, B. J., Albertorio, F., Cremer, P. S. (2007) Separation of Membrane-Bound Compounds by Solid-Supported Bilayer Electrophoresis, J Am Chem Soc 129, 8072-8073. bttps: / doi.org/ 10.1021/ja0720816

38. Tanaka, M., Hermann, J., Haase, I., Fischer, M., Boxer, S. G. (2007) Frictional Drag and Electrical Manipulation of Recombinant Proteins in Polymer-Supported Membranes, Langmuir 23, 5638-5644. https:/ doi.org/10.1021/ la0628219

39. Monson, C. F., Pace, H. P., Liu, C., Cremer, P. S. (2011) Supported Bilayer Electrophoresis under Controlled Buffer Conditions, Anal Chem 83, 2092-2096. https://doi.org/10.1021/ac1028819

40. Liu, C., Monson, C. F., Yang, T., Pace, H., Cremer, P. S. (2011) Protein Separation by Electrophoretic-Electroosmotic Focusing on Supported Lipid Bilayers, Anal Chem 83, 7876-7880. https:// doi.org/10.1021/ac201768k

41. Jönsson, P., Beech, J. P., Tegenfeldt, J. O., Höök, F. (2009) Shear-Driven Motion of Supported Lipid Bilayers in Microfluidic Channels, J Am Chem Soc 131, 5294-5297. https:/ / doi.org/10.1021/ja809987b

42. Jönsson, P., Beech, J. P., Tegenfeldt, J. O., Höök, F. (2009) Mechanical Behavior of a Supported Lipid Bilayer under External Shear Forces, Langmuir 25, 6279-6286. https://doi.org/10.1021/la8042268

43. Hoopes, M. I., Faller, R., Longo, M. L. (2011) Lipid Domain Depletion at Small Localized Bends Imposed by a Step Geometry, Langmuir 27, 2783-2788. bttps:// doi.org/10.1021/la104504p

44. Motegi, T., Nabika, H., Fu, Y., Chen, L., Sun, Y., Zhao, J., Murakoshi, K. (2014) Effective Brownian Ratchet Separation by a Combination of Molecular Filtering and a Self-Spreading Lipid Bilayer System, Langmuir 30, 7496-7501. bttps:// doi.org/10.1021/la500943k

45. Neumann, J., Hennig, M., Wixforth, A., Manus, S., Rädler, J. O., Schneider, M. F. (2010) Transport, Separation, and Accumulation of Proteins on Supported Lipid Bilayer, Nano Lett 10, 2903-2908. https:// doi.org/10.1021/nl100993r

46. Wagner, M. L., Tamm, L. K. (2000) Tethered Polymer-Supported Planar Lipid Bilayers for Reconstitution of Integral Membrane Proteins: Silane-Polyethyleneglycol-Lipid as a Cushion and Covalent Linker, Biophys J 79, 1400-1414. bttps:// doi.org/10.1016/S0006-3495(00)76392-2

47. Wagner, M. L., Tamm, L. K. (2001) Reconstituted Syntaxin1A/SNAP25 Interacts with Negatively Charged Lipids as Measured by Lateral Diffusion in Planar Supported Bilayers, Biophys J 81, 266-275. https:/ / doi.org/10.1016/S0006$3495(01) 75697-4$

48. Goennenwein, S., Tanaka, M., Hu, B., Moroder, L., Sackmann, E. (2003) Functional Incorporation of Integrins into Solid Supported Membranes on Ultrathin Films of Cellulose: Impact on Adhesion, Biophys J 85, 646-655. bttps: / / doi.org/ 10.1016/S0006-3495(03)74508-1

49. Purrucker, O., Förtig, A., Jordan, R., Tanaka, M. (2004) Supported Membranes with Well-Defined Polymer TethersIncorporation of Cell Receptors, ChemPbysChem 5, 327-335. https:// doi.org/10.1002/cphc.200300863 
50. Purrucker, O., Förtig, A., Jordan, R., Sackmann, E., Tanaka, M. (2007) Control of Frictional Coupling of Transmembrane Cell Receptors in Model Cell Membrnes with Linear Polymer Spacers, Phys Rev Lett 98, 078102. bttps:// doi.org/10.1103/PhysRevLett.98.078102

51. Richards, M. J., Hsia, C.-Y., Singh, R. R., Haider, H., Kumpf, J., Kawate, T., Daniel, S. (2016) Membrane Protein Mobility and Orientation Preserved in Supported Bilayers Created Directly from Cell Plasma Membrane Blebs, Langmuir 32, 29632974. bttps:/ / doi.org/ 10.1021/ acs.langmuir.5b03415

52. Hertrich, S., Stetter, F., Rühm, A., Hugel, T., Nickel, B. (2014) Highly Hydrated Deformable Polyethylene Glycol-Tethered Lipid Bilayers, Langmuir 30, 9442-9447. https:/ / doi.org/10.1021/ la4045804

53. Kam, L., Boxer, S. G. (2003) Spatially Selective Manipulation of Supported Lipid Bilayers by Laminar Flow: Steps Toward Biomembrane Microfluidics, Langmuir 19, 1624-1631. https:/ / doi.org/ 10.1021/ la0263413

54. Lee, S. C., Knowles, T. J., Postis, V. L. G., Jamshad, M., Parslow, R. A., Lin, Y., Goldman, A., Sridhar, P., Overduin, M., Muench, S. P., Dafforn, T. (2016) A Method for Detergent-free isolation of Membrane Protein from its Local Lipid Environment, Nature Prot 11, 1149-1162. https:/ / doi.org/ 10.1038/ nprot.2016.070

55. Simon, K. S., Pollock, N. L., Lee, S. C. (2018) Membrane protein nanoparticles: the shape of things to come, Biochem Soc Trans 46, 1495-1504. https:// doi.org/10.1042/BST20180139

56. Hope, M. J., Bally, M. B., Webb, G., Cullis, P. R. (1985) Production of Large Unilamellar Vesicles by a Rapid Extrusion Procedure: Characterization of Size Distribution, Trapped Volume and Ability to Maintain a Membrane Potential, Biochim Biophys Acta 812, 55-65. https:/ / doi.org/10.1016/0005-2736(85)90521-8

57. Mayer, L. D., Hope, M. J., Cullis, P. R. (1986) Vesicles of Variable Sizes Produced by a Rapid Extrusion Procedure, Biochim Biophys Acta 858, 161-168. https:/ / doi.org/10.1016/0005-2736(86)90302-0

58. Yang, T., Jung, S.-Y., Mao, H., Cremer, P. S. (2001) Fabrication of Phospholipid Bilayer-Coated Microchannels for On-Chip Immunoassays, Anal Chem 73, 165-169. https:// doi.org/10.1021/ac000997o

59. Zhu, L., Gregurec, D., Reviakine, I. (2013) Nanoscale Departures: Excess Lipid Leaving the Surface during Supported Lipid Bilayer Formation, Langmuir 29, 15283-15292. https:/ / doi.org/10.1021/ la401354j

60. Pace, H. P., Sherrod, S. D., Monson, C. F., Russell, D. H., Cremer, P. S. (2013) Coupling Supported Lipid Bilayer Electrophoresis with MALDI-MS Imaging, Anal Chem 85, 6047-6052. bttps:/ / doi.org/10.1021/ac4008804

\section{ABOUT STUDENT AUTHORS}

Michael J. Ornstead graduated in April 2019 and is currently working on his MD at the University of Utah.

Ruth Hunter graduated in April 2020 and is planning to attend medical school.

Mason L. Valentine graduated in April 2015 and is getting a $\mathrm{PhD}$ at the University of Texas, Austin.

Cameron Cooper graduated in April 2015, earned a degree as a physician assistant, and is currently working in the field.

Stephen K. Smith graduated in April 2019 and is currently working on a dental degree at the University of Utah.

\section{PRESS SUMMARY}

Cell membranes, the "bags" that hold our cells together, are composed of lipids and membrane proteins. Membranes are essential parts of cells, with many different processes occurring at or across them. Membranes are also difficult to study biochemically, as they have both water and oil soluble parts. Because of this, several methods to study membranes are being developed around the world. Many of them use supported lipid bilayers, flat sheets of membrane material sitting on a solid support such as glass. The authors have developed a method to study lipids in a supported lipid bilayer from the glass and put the material into solution. This is accomplished by flowing solution over the lipid sheet at high speeds. This could help with efforts to learn more about membranes, particularly membrane proteins, as it may allow supported lipid bilayers to be converted into more cell-like structures. 